\title{
Hyperosmotic versus Hypoosmotic Stress in Plants
}

Veronika Hýskova* and Helena Ryslava

Department of Biochemistry, Charles University in Prague, Czech Republic

*Corresponding author: Veronika Hýsková, Department of Biochemistry, Charles University in Prague, Czech Republic, Tel: +420221951595; E-mail: veronika.hyskova@natur.cuni.cz

Received date: February 02, 2018; Accepted date: February 23, 2018; Published date: March 02, 2018

Copyright: ( 2018 Hýsková V, et al. This is an open-access article distributed under the terms of the Creative Commons Attribution License, which permits unrestricted use, distribution, and reproduction in any medium, provided the original author and source are credited.

\begin{abstract}
The balance of water between the cell and its environment is crucial to all organisms, but especially for root plant cells that are exposed to extrinsic condition directly. High salt concentration in soil leads to loose of water from the plant cell (hyper-osmotic stress). Sufficient supply with water causes that the plant cell is turgid (i.e. increases its volume, the cell membrane experiences turgor pressure from the cell interior against the resistance of the cell wall), which is healthy state for most plants. However, upon repeated flooding (e.g. in a tropical rain-forest climate), the plants may experience a prolonged hypo-osmotic stress. Whereas the hyper-osmotic stress is one of the most studied abiotic stress factor, hypo-osmotic is at the edge of scientists' interest, still considered as "physiological conditions". However, both types of osmotic stress are characterized by involvement of stress sensing, oxidative burst, and signal transduction. In this study, sensors of osmotic stress (mechano-sensitive ion channels), osmolytes, and processes followed hypo- and hyper-osmotic stress are emphasized, although hypo-osmotic stress in comparison to hyper- have to be largely studied.
\end{abstract}

Keywords: Hypo-osmotic stress; Hyperosmotic stress; Mechanosesnsitive channels; Osmolytes; NADP-dependent enzymes

\section{Introduction}

The balance of water between the cell and its environment is crucial to all organisms. It is well known that during process called osmosis water diffuses across the membrane from the region of lower solute concentration to that of higher solute concentration until the solute concentrations on both sides of the membrane are equal. Extremely hypertonic environment leads to plasmolysis (loose of water from a cell to its surroundings, which cause that a cell shrivels, in the plant cell the plasma membrane pulls away from the cell wall), whereas in hypotonic environment a cell swells as water enters [1]. Animal multicellular cells are protected from osmotic challenges by excretion organs, which are able to secrete or reabsorb ions so that isotonic environment for cells is established and maintained [2].

\section{The role of cell wall}

The strategy used by animals is not possible for bacteria, fungi, and plants that have to cope with strong variations of osmotic conditions [2]. On the other hand, these organisms possess a strong cell wall. Whereas bacteria cell wall is composed of peptidoglycan murein (polymer of $\mathrm{N}$-acetylmuramic and $\beta$-N-acetylglucosamine residues) and fungi cell wall is formed from chitin (an unbranched polysaccharide consisting of $\beta-\mathrm{N}$-acetylglucosamine residues) and/or $\beta$-glucan [1]. Plant cell wall is comprised of cellulose microfibrils (an unbranched polymers consisting of $\mathrm{D}$-glucose molecules connected by $\beta$-1,4-glycosidic linkages). These cellulose microfibrils have an unusually high tensile strength, they are very resistant to chemical and biological degradation and their crystalline regions are impermeable to water [3]. The cellulose microfibrils are interconnected by hemicellulose tethers (branched polysaccharides consisted of variety of saccharides in addition to D-glucose) embedded in a gel of pectin (a mixture of polymers from sugar acids, such as D-galacturonic acid). The polymers of phenylpropane derivatives: lignin, suberin, and cutin and also waxes further contribute to the strength of plant cell walls, which protect the cell from the adverse effects from the environment [3-5].

\section{Sensing of osmotic stress: Mechano-sensitive ion channels}

Surface receptors incorporated in the membrane bilayer play a key role in receiving signals from the environment outside. Membrane ion channels, which perceive the signals typically in the form of chemical substances (such as hormones and neurotransmitters) constitute the basic class of the surface receptors. Yet another form of the signal, which affects the surface receptors, is the mechanical stimulus including pressure, shear stress, and osmolarity [6].

Mechano-sensitive (MS) ion channels are transmembrane proteins that directly couple mechanical stimuli to ion flux [7]. Mechanosensation is present in all the species ranging from bacteria to mammalians [8]. In humans (or mammalians) it has been reported that MS channels are involved in several important physiological functions such as sensation of tactile stimulus, pain, hearing, proprioception, synaptogenesis, regulating of cell volume and heart rate [8]. On the contrary malfunction of MS channels confer pathological processes e.g. arrhythmia, muscular dystrophy, pulmonary hypertension, polycystic kidney disease, and tumor progression. Non-specific cation channels called the transient receptor potential (TRP) channels, which are localized in sensory neurons, cardiomyocytes, renal epithelial cells, osteoblasts, inner hair cells etc. represent large group of human MS channels $[6,8]$.

Escherichia coli MscS is one of the best understood MS ion channels in any system. It is as essentially non-selective ion channel, gated directly by membrane tension, with a large conductance. The classic function of $E$. coli $\mathrm{MscS}$ is to serve as an osmotic safety valve 
protecting cells from rupture during extreme hypo-osmotic downshock [7].

In plant systems, MS ion channels are widely distributed across multiple species, cell types, and intracellular compartments [7]. Plant MS ion channels have been proposed to play a wide array of roles, from perception of touch and gravity to the osmotic homeostasis of intracellular organelles. Three families of plant MS ion channels were identified: the MscS-like (MSL), Mid1-complementing activity (MCA), and two pore Potassium (TPK) families. These three families are still likely to represent only a fraction of the MS ion channel diversity in plants [9]. There are 10 MSL proteins in Arabidopsis, most of which are predicted to localize in plasma membrane, but also to the inner membrane of plastids and mitochondria [7]. MSLs as essentially nonselective ion channels are important to organelle osmoregulation and likely play complex roles [9].

Despite a much effort only a few putative osmotic sensors have been identified. One of the MS channels (which fully meets the criteria for a mechanoreceptor) in Arabidopsis: MSL8 is required for pollen to survive hypo-osmotic shock during hydratation suggesting MSL8 as a sensor of hypo-osmotic stress-induce membrane tension $[5,7,10]$. It was proposed that the role of MSL8 consist in controlling turgor during pollen hydratation, germination, and tube growth. Disruption of MSL8 results in high rates of bursting during pollen hydratation and germination. Although excess turgor after hydratation leads to germination at a rate higher than wild type, frequent bursting leads to an overall loss of fertility. On the contrary, overexpression of MSL8 from the pollen-specific strong promoter cause that pollen grains survive hydratation but are unable to maintain the threshold turgor pressure required for pollen germination and pollen tube elongation [7].

\section{Osmolytes}

One acclimation strategy to osmotic and/or salt stress conditions relies on the massive accumulation of low molecular compounds, so called compatible solutes or osmoprotectants. The major role of these metabolites is increase the ability of cells to retain water without disturbing of normal cellular functions. Osmolytes not only balance osmotic potential gradients, but also directly protect critical macromolecules from damage. The compatible solutes are small, nontoxic molecules that are characterized by high water solubility (allowing their accumulation in high amounts) and usually no net charge to avoid impact on membrane potential. The metabolites which act as compatible solutes are different among various species of plants. Osmolytes can be grouped into a few chemical classes, namely ammonium compounds (polyamines, betaines), sugars (trehalose), sugar alcohols (manitol, sorbitol, ononitol), and amino acids and their derivatives (proline). [11-13]. Glycine betaine (N,N,Ntrimethylglycine) is widely distributed osmolyte protecting cells from salt stress and simultaneously stabilizing photosynthetic apparatus, sugars provide excellent protection to cell membranes (hydroxyl groups of certain sugars allows direct replacement of water at the membrane surface, and polyols are superior in water solubility and osmotic effects [11-13].

\section{Hypo-osmotic stress in plants, much less studied but followed by defense responses}

Upon sufficient supply with water (surrounding hypotonic solution) the plant cell increases its volume, the cell membrane experiences considerable pressure from the cell interior against the resistance of the cell wall (i.e. turgor pressure). At this point the cell is turgid, which is healthy state for most plants $[1,2]$.

However, in inundation areas and in a tropical rain-forest climate (dominated by monsoon rainfall), the plants may experience a prolonged hypo-osmotic stress [14]. Hypo-osmotic stress may occur transiently when dry soil is rapidly rewetted, and chronically upon repeated flooding [4]. It has been shown that hypo-osmotic stress similarly as hyper-osmotic induces together with ion fluxes an oxidative burst $[5,15]$. Even though similar signaling pathways are involved in both types of osmotic stresses, only a little attention is devoted to the study of hypo-osmotic stress defense responses.

Although the hypo-osmotic stress is at the edge of interest, still considered as "physiological conditions", it can increase membrane fluidity and tonoplast proton pumps activity [16] and also induce reactive oxygen species generation mediated by NADPH-oxidases [17]. During hypo-osmotic stress the oxidative response depends on extracellular $\mathrm{Ca}^{2+}$ signals and phosphorylation events. Such stress signaling regulates proteins critical for metabolic and gene-expression reprogramming to restore again water homeostasis and cellular stability under stress conditions [5,15]. In addition, the movement of water extracted from the soil by plant's roots up the plant through the vascular system also mediates root-to shoot movement of nutrients, hormones, and developmental signals [4] however excess of water (e.g. flooding with pure e.g. rainfall water) can represent further nutrient deprivation for plant.

\section{Hyper-osmotic salt stress, one of the most studied abiotic stress}

The hyper-osmotic stress induced by salt (mostly $\mathrm{NaCl}$ ) is probably the most studied type of abiotic stress (maybe due to the proportion of salt-affected irrigated land in various countries ranges from 9 to $34 \%$, with a world average of 20\%) [18]. A hundreds of thousands of studies were done to elucidate salt stress-induced physiological, biochemical and molecular changes on transcriptional, translational, and posttranslational level (e.g. reviews: [18-21]. -Both the concentration gradient and voltage differential across plasma membrane favor the passive entry of $\mathrm{Na}^{+}$from the soil solution into the cytoplasm of root cells. Responsible for influx of $\mathrm{Na}^{+}$into cells are mainly: Non-Selective Cation Channels (NSCCs), Cyclic-Nucleotide Gated Channels (CNGCs), and High affinity potassium (K) Transporters (HKT) [22]. High salt levels in cells cause ion toxicity (mainly $\mathrm{Na}^{+}$) and ion imbalance, hyperosmotic stress, and secondary stresses such as oxidative damage and nutrient imbalance [5]. The $\mathrm{Na}^{+}$ions are toxic due to their unfavorable effect on $\mathrm{K}^{+}$nutrition $\left(\mathrm{K}^{+}\right.$as essential nutrient in plants) and effect on cytosolic enzymes activities. Increased soil salt concentration decrease soil water potential and thus a decrease the ability of a plant to take up water, i.e. osmotic stress [20,23,24]. It leads to a decreased turgor, closed stomata and therefore a reduced photosynthesis and inhibition of plant growth. When the difference in the water potential cannot be compensated, the dehydratation of the cell occurs [21]. In addition, salinity-induced osmotic effects alter general metabolic processes and enzymatic activities leading to excessive accumulation of reactive oxygen species [25]. Up-regulated production of reactive oxygen species (hydrogen peroxide, superoxide radical, singlet oxygen, and hydroxyl radical etc.) causes phytotoxic reactions in the form of lipid peroxidation, protein degradation, and DNA mutation. Mostly membrane injury induced by salt stress is related to an enhanced production of highly toxic reactive oxygen 
Page 3 of 4

species [26]. Therefore, rapid mobilization of antioxidant system in the form of defense compounds and antioxidant enzymes is crucial for plant defense against salt stress. Main defense mechanisms towards salt stress preventing harmful effects are active $\mathrm{Na}^{+}$efflux, restriction of $\mathrm{Na}$ + influx, sequestration of $\mathrm{Na}^{+}$ions in vacuole, and the synthesis of molecules having a protective function [19-21,27,28].

For salt stress signaling plants use a calcium dependent protein kinase pathway known as SOS (salt overly sensitive), composed of SOS1, which is $\mathrm{Na}^{+} / \mathrm{H}^{+}$antiporter at the plasma membrane. SOS1 is expressed in root epidermal cells and xylem parenchyma cells, so that activated SOS1 can extrude $\mathrm{Na}^{+}$in the soil solution and load $\mathrm{Na}^{+}$into the xylem for long-distance transport to leaves [5]. Sequestration of toxic $\mathrm{Na}^{+}$ions in vacuole is mediated by vacuolar $\mathrm{Na}^{+} / \mathrm{H}^{+}$antiporter [22]. The accumulation of organic osmolytes (mentioned above), such as proline, glycine betaine, sugar alcohols (mannitol, sorbitol) polyamines and proteins from the late embryogenesis abundant (LEA) superfamily, plays a key role in the low intracellular osmotic potential of plants $[18,20]$. To cope with plant stress, plants have evolved complex defense responses that involve stress sensing, salt-responsive signal transduction (dependent or independent on intracellular $\mathrm{Ca}^{2+}$, kinases, hormones such as abscisic acid, jasmonate; reactive oxygen species etc.), and activation of a number of stress-related genes and metabolites. Changes at the cellular, organ, and whole-plant levels are helpful in alleviating of adverse effect of the stress [23,29,30]. Proteome changes in both salt-tolerant plants (halofytes) and salt -sensitive plants (glycophytes, crops) include reactive oxygen species-scavenging enzymes and enzymes involved in biosynthesis of compatible solutes (osmolytes such as glycine betaine). Salt stress in glycophytes also increased abundance of enzymes involved in glycolysis or biosynthesis of fatty acids, nucleotides, and saccharides (which indicates a higher need for energy) [24].

One of the main finding of metabolite analysis of Arabidopsis thaliana cell cultures under salt stress was enhanced methylation accompanied by induction of aromatic acid biosynthesis that led to salt-promoted production of lignin biosynthesis, probably for cell wall strengthening [23].

\section{Increased activity of NADP-dependent enzymes accompanied both types of osmotic stress}

Research from of our laboratory documented the comparison of defense responses to both types of stress (hyper- and hypo-osmotic). We have found out, which enzymes are important for defense responses and for maintaining of cellular metabolism. NADPH represents an indispensable compound for biosynthetic reactions (such as biosynthesis of osmotically active compounds or biosynthesis of fatty acids for damage membranes repairing), antioxidant systems (NADPH as cofactor of antioxidant enzymes), and for enzymes involved in regulation (NADPH-dependent thioredoxin reductase). As the ratio $\mathrm{NADP}^{+} / \mathrm{NADPH}$ in plants influences redox reactions, the control of cell redox homeostasis is important for balancing metabolic processes and redox-dependent signalling.

NADPH is produced mainly in dark by enzymes from oxidative pentose phosphate pathway (glucose-6-phosphate dehydrogenase, 6phosphogluconate dehydrogenase) but also other enzymes represent an alternative source of NADPH in stressed plants (e.g. NADPisocitrate dehydrogenase, NADP-malic enzyme, NADP-glutamate dehydrogenase, and non-phosphorylating NADP-glyceraldehyde-3phosphate dehydrogenase) [31]. Various functions of NADP- dependent enzymes during abiotic stress were suggested e.g. the involvement of G6PDH in regulation of $\mathrm{Na}^{+} / \mathrm{H}^{+}$antiporter through providing of NADPH for plasma membrane NADPH-oxidase as an adaptation to salt stress [32].

Our study with cucumber (Cucumis sativa L.) seedlings indicated that after 3 days of stress the consequences of salt stress $(100 \mathrm{mM}$ $\mathrm{NaCl}$ ) were worse than hypo-osmotic stress (caused by distilled water) judged by determination of relative water content, $\mathrm{Na}^{+}, \mathrm{K}^{+}$ions content, phenolic compounds, Rubisco and Heat shock protein 70 contents). In these parameters hypo-osmotically stressed plants were mostly comparable with plants grown in soil with exception of phenolic compounds (the 2nd day of hypo-osmotic stress enhanced content of total phenolic compounds and flavonoids was determined). On the other hand, relative water content in salt stressed leaves decreased up to $60 \% ; \mathrm{Na}^{+}$ion content in salt-stressed roots was 15 -fold higher than in roots of plant grown in soil and on the contrary $\mathrm{K}^{+}$was almost undetectable in salt stress roots. The amount of Rubisco content was in salt-stressed leaves decreased and protein related to abiotic stress heat shock protein 70 increased [33]. Hypo-osmotic stress was accompanied with the more pronounced changes in specific activities of NADP-dependent enzymes (such as glucose-6-phosphate dehydrogenase, NADP-malic enzyme, NADP-isocitrate dehydrogenase, non-phosphorylating glyceraldehyde-3-phosphate dehydrogenase, and shikimate dehydrogenase) in leaves. The specific activity of glucose-6-phosphate dehydrogenase was the most enhanced, 7.4-fold and 3.5-fold in hypo-osmotically and salt stress, respectively [33]. Salt stress induce at the beginning of experiment (when the extension of plant damage was lesser) activity of NADPgalactose-1-dehydrogenase and ribose-1-dehydrogenase in both leaves and roots. Therefore, we assume that during milder stress increased demands of NADPH can be replenished by a wide variety of NADPdependent enzymes [31,33,34].

Further experiments are needed to elucidate all the differences and the similarities of defense responses in important crop plants exposed to hyper- and hypo-osmotic stress. Such findings could be helpful in improving of plant cell defense against osmotic stress.

\section{Conclusion}

Although the direction of water flow during hypo- and hyperosmotic is opposite, both these types of stress share similar features, both affect osmotic sensors, caused enhance production of reactive oxygen species, and induce signal transductions. Which genes and metabolic pathways are affected by these signaling is largely studied during salt (hyper-osmotic stress) but little is known about hypoosmotic stress. To know all the details about metabolic rearrangements and acclimatization to both types of osmotic stress further experiments are needed.

\section{References}

1. Cambell NA, Reece JB (2008) Biology (8thedn), Pearson Benjamin Cummings, San Francisco, Boston, New York, Cape Town, Hong Kong, London, Madrid, Mexico City, Montreal, Munich, Paris, Singapore, Sydney, Tokyo, Toronto.

2. Liu Q, Qiao F, Ismail A, Chang X, Nick P (2013) The plant cytoskeleton controls regulatory volume increase. Biochim Biophys Acta 1828: 2111-2120.

3. Heldt HW, Piechulla B, Heldt F (2011) Plant Biochemistry-Translation of the 4th German edn, Elsevier Academic Press, Amsterdam, Boston, 
Citation: Hýskova V, Ryslava H (2018) Hyperosmotic versus Hypoosmotic Stress in Plants. Biochem Anal Biochem 7: 1000e170. doi:

Page 4 of 4

Heildelberg, London, New York, Oxford, Paris, San Diego, San Francisco, Singapore, Sydney, Tokyo.

4. Haswell ES, Verslues PE (2015) The ongoing search for the molecular basis of plant osmosensing. J Gen Physiol 145: 389-394.

5. Zhu JK (2016) Abiotic stress signaling and responses in plants. Cell 167: 313-324.

6. Takahashi K, Matsuda Y, Naruse K (2016) Mechanosensitive ion channels. AIMS Biophysics 3: 63-74.

7. Basu D, Haswell ES (2017) Plant mechanosensitive ion channels: an ocean of possibilities. Curr Opin Plant Biol 40: 43-48.

8. Purali N (2017) Mechanosensitive ion channels. J Mol Sci 1: 1-4.

9. Hamilton ES, Schlegel AM, Haswell ES (2015) United in diversity: mechanosensitive ion channels in plants. Annu Rev Plant Biol 66: 113-137.

10. Hamilton ES, Jensen GS, Maksaev G, Katims A, Sherp AM, et al. (2015) Mechanosensitive channel MSL8 regulates osmotic forces during pollen hydration and germination. Science 350: 438-441.

11. Hagemann M, Pade N (2015) Heterosides: compatible solutes occurring in prokaryotic and eukaryotic phototrophs. Plant Biol (Stuttg) 17: 927-934.

12. Singh M, Kumar J, Singh S, Singh VP, Prasad SM (2015) Roles of osmoprotectants in improving salinity and drought tolerance in plants: a review. Rev Environ Sci Biotechnol 14: 407-426.

13. Wani SH, Singh NB, Haribhushan A, Mir JI (2013) Compatible solute engineering in plants for abiotic stress tolerance - role of glycine betaine. Curr Genomics 14: 157-165.

14. Teo SS, Ho CL, Teoh S, Rahim RA, Phang SM (2009) Transcriptomic analysis of Gracilaria changii (Rhodophyta) in response to hyper- and hypoosmotic stresses. J Phycol 45: 1093-1099.

15. Cazale AC, Droillard MJ, Wilson C, Heberle-Bors E, Barbier-Brygoo H, et al. (1999) MAP kinase activation by hypoosmotic stress of tobacco cell suspensions: towards the oxidative burst response? Plant J 19: 297-307.

16. Ozolina NV, Kolesnikova EV, Nurminsky VN, Nesterkina IS, Dudareva LV, et al. (2011) Redox-dependence of transport activity of tonoplast proton pumps: Effects of nitrogen oxide exposure in the course of ontogenesis and of hypo- and hyper-osmotic stress. Biologicheskie Membrany 28: 284-289.

17. Kurusu T, Nishikawa D, Yamazaki Y, Gotoh M, Nakano M, et al. (2012) Plasma membrane protein OsMCA1 is involved in regulation of hypoosmotic shock-induced $\mathrm{Ca} 2+$ influx and modulates generation of reactive oxygen species in cultured rice cells. BMC Plant Biol 12: 1-15.

18. Parihar P, Singh S, Singh R, Singh VP, Prasad SM (2014) Effect of salinity stress on plants and its tolerance strategies: a review. Environ Sci Pollut Res Int 22: 4056-4075.

19. Abbasi H, Jamil M, Haq A, Ali S, Ahmad R, et al. (2016) Salt stress manifestation on plants, mechanism of salt tolerance and potassium role in alleviating it: a review. Zemdirbyste-Agriculture 103: 229-238.
20. Deinlein U, Stephan AB, Horie T, Luo W, Xu G, et al. (2014) Plant salttolerance mechanisms. Trends Plant Sci 19:371-379.

21. Isayenkov SV (2012) Physiological and molecular aspects of salt stress in plants. Cytol Genet 46: 302-318.

22. Plett D, Moller Skumsager I (2010) Na+ transport in glycophytic plants: what we know and would like to know. Plant Cell Environ 33: 612-626.

23. Jorge TF, Rodrigues JA, Caldana C, Schmidt R, van Dongen JT, ThomasOates J, Antonio C (2015) Mass spectrometry-based plant metabolomics: Metabolite responses to abiotic stress. Mass Spectrom Rev 35: 620-649.

24. Kosova K, Vitamvas P, Prasil IT, Renaut J (2011) Plant proteome changes under abiotic stress-contribution of proteomics studies to understanding plant stress response. J Proteomics 74: 1301-1322.

25. Farooq M, Hussain M, Wakeel A, Siddique K (2015) Salt stress in maize: effects, resistance mechanisms, and managments. A review. Agron Sustain Dev 35: 461-481.

26. Nawaz K, Hussain K, Majeed A, Khan F, Afghan S, et al. (2010) Fatality of salt stress to plants: Morphological, physiological and biochemical aspects. Afr J Biotechnol 9: 5475-5480.

27. Shi H, Ishitani M, Kim C, Zhu JK (2000) The Arabidopsis thaliana salt tolerance gene SOS1 encodes a putative $\mathrm{Na}+\mathrm{H}+$ antiporter. P Natl Acad Sci USA 97: 6896-6901.

28. Hauser F, Horie T (2010) A conserved primary salt tolerance mechanism mediated by HKT transporters: a mechanism for sodium exclusion and maintenance of high $\mathrm{K}+/ \mathrm{Na}+$ ratio in leaves during salinity stress. Plant Cell Environ 33: 552-565.

29. Zhang H, Han B, Wang T, Chen S, Li H, et al. (2011) Mechanisms of plant salt response: insights from proteomics. J Proteome Res 11: 49-67.

30. Golldack D, Li C, Mohan H, Probst N (2014) Tolerance to drought and salt stress in plants: unraveling the signaling networks. Front Plant Sci

31. Doubnerova V, Ryslava H (2012) NADP-dependent enzymes and abiotic stress. in: N. Haryana, and S. Punj, (Eds.), Abiotic Stress New Research, Nova Science Publishers, pp. 57-97.

32. Li J, Chen G, Wang X, Zhang Y, Jia H, et al. (2011) Glucose-6-phosphate dehydrogenase-dependent hydrogen peroxide production is involved in the regulation of plasma membrane $\mathrm{H}+$-ATPase and $\mathrm{Na}+\mathrm{H}+$ antiporter protein in salt-stressed callus from Carex moorcroftii. Physiol Plant 141: 239-250.

33. Hyskova V, Pliskova V, Cerveny V, Ryslava H (2017) NADP-dependent enzymes are involved in response to salt and hypoosmotic stress in cucumber plants. Gen Physiol Biophys 36: 247-258.

34. Doubnerova V, Ryslava H (2011) What can enzymes of C4 photosynthesis do for C3 plants under stress? Plant Sci 180: 575-583. 